\title{
Influencia de la zeolita en la emisión de óxido nitroso y uso eficiente de nutrientes en maíz dulce
}

\section{Influence of zeolite on the nitrous oxide emission and efficient use of nutrients in sweet corn}

CLAUDIA MARTÍNEZ-HERRERA1,3

YINA JAZBLEIDI PUENTES-PÁRAMO ${ }^{2}$

JUAN CARLOS MENJIVAR-FLORES²

Cultivo de maíz dulce.

Foto: C. Martínez-Herrera

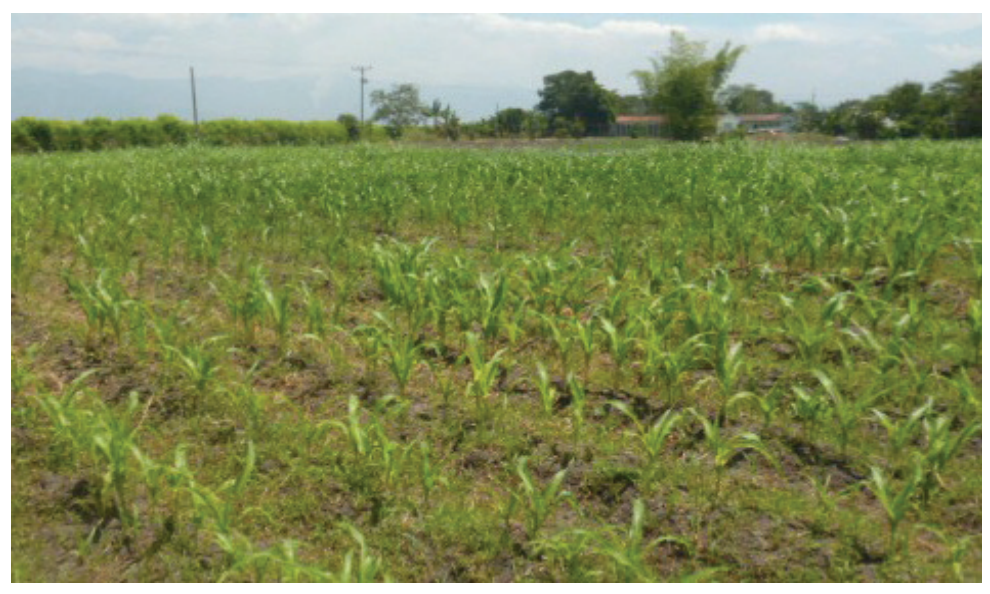

\section{RESUMEN}

La investigación tuvo como objetivo determinar la influencia de zeolita (clinoptilolita) en la emisión de óxido nitroso $\left(\mathrm{N}_{2} \mathrm{O}\right)$ y el uso eficiente de nutrientes N-P-K en el cultivo de maíz dulce (Zea mays L.), utilizando la técnica isotópica de ${ }^{15} \mathrm{~N} 2,5 \%$ en exceso. Se utilizó un diseño en bloques completos al azar con cinco tratamientos y cuatro repeticiones, se emplearon dosis de 236,75 y $192 \mathrm{~kg} \mathrm{ha}^{-1}$ para N, P y K respectivamente, mezclados con $0,70,140$ y $210 \mathrm{~kg} \mathrm{ha}^{-1}$ de zeolita. La emisión de $\mathrm{N}_{2} \mathrm{O}$ se estimó mediante cámara estática cerrada a los $15,25,36,45,56,65$ y 76 días después de la siembra del cultivo. Se determinó el uso eficiente de nutrientes mediante la eficiencia agronómica y de recuperación del fertilizante para N-P-K. El flujo de $\mathrm{N}_{2} \mathrm{O}$ y las eficiencias de uso de nutrientes por tratamiento mostraron diferencias altamente significativas $(P \leq 0,01)$; el mayor flujo de $\mathrm{N}_{2} \mathrm{O}$ se presentó en el testigo y menor en el T4 (210 kg ha-1 zeolita, $236 \mathrm{~kg} \mathrm{ha}^{-1}$ nitrógeno, $75 \mathrm{~kg} \mathrm{ha-1}$ fósforo $\mathrm{P}_{2} \mathrm{O}_{5}$ y $192 \mathrm{~kg} \mathrm{ha}^{-1}$ potasio $\mathrm{K}_{2} \mathrm{O}$ ), contrastante con la mayor eficiencia de uso de nutrientes en el T4 excepto para nitrógeno. Esto evidencia la contribución de la zeolita en la disminución de la emisión de $\mathrm{N}_{2} \mathrm{O}$ e incremento del rendimiento.

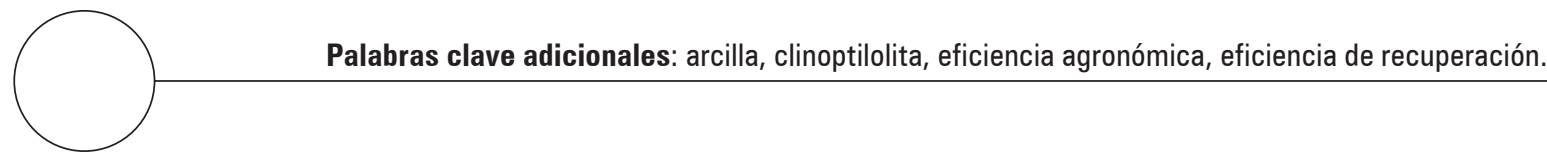

\footnotetext{
Facultad de Ciencias Agropecuarias, Programa de Maestría en Ciencias Agrarias, Universidad Nacional de Colombia, Palmira (Colombia). ORCID Martínez-Herrera, C.: 0000-0003-2617-3041

2 Facultad de Ciencias Agropecuarias, Universidad Nacional de Colombia, Palmira (Colombia). ORCID Puentes-Páramo, Y.J.: 0000-0002-3784-9687; ORCID Menjivar-Flores, J.C.: 0000-0002-0985-7778

3 Autor para correspondencia. claudia.martinez@cvc.gov.co
} 


\section{ABSTRACT}

This research aimed to determine the influence of zeolite (clinoptilolite) on the emission of nitrous oxide $\left(\mathrm{N}_{2} \mathrm{O}\right)$ and the efficient use of N-P-K nutrients in sweet corn (Zea mays L.) cultures using the ${ }^{15} \mathrm{~N}$ isotopic technique, $2.5 \%$ in excess. A complete randomized block design with five treatments and four replicates was used; doses of 236,75 and $192 \mathrm{~kg} \mathrm{ha}^{-1}$ were used for N, P and K, respectively, mixed with 0, 70, 140 and $210 \mathrm{~kg} \mathrm{ha}^{-1}$ of zeolite. The emission of $\mathrm{N}_{2} \mathrm{O}$ was estimated with a closed static chamber at $15,25,36,45,56,65$ and 76 days after sowing the crop. The efficient nutrient use was determined with the agronomic and recovery fertilizer efficiency of N-P-K. $\mathrm{N}_{2} \mathrm{O}$ flow and nutrient use efficiencies by treatment showed highly significant differences $(P \leq 0.01)$; the highest $\mathrm{N}_{2} \mathrm{O}$ flux was found in the control and the lowest was in T4 $\left(210 \mathrm{~kg} \mathrm{ha}^{-1}\right.$ zeolite, $236 \mathrm{~kg} \mathrm{ha}^{-1}$ nitrogen, $75 \mathrm{~kg} \mathrm{ha}^{-1}$ phosphorus $\mathrm{P}_{2} \mathrm{O}_{5}$ and $192 \mathrm{~kg} \mathrm{ha}^{-1}$ potassium $\mathrm{K}_{2} \mathrm{O}$ ) as contrasted with the greater nutrient use efficiency in $\mathrm{T} 4$, except for nitrogen. This showed the contribution of zeolite in the reduction of $\mathrm{N}_{2} \mathrm{O}$ emissions and in yield increases.

Additional key words: agronomic efficiency, clay, clinoptilolite, recovery efficiency.

Fecha de recepción: 21-06-2017 Aprobado para publicación: 30-09-2017

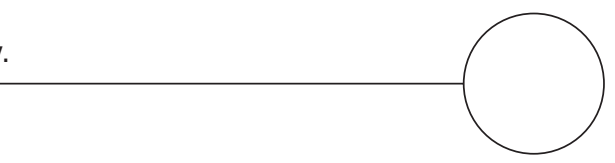

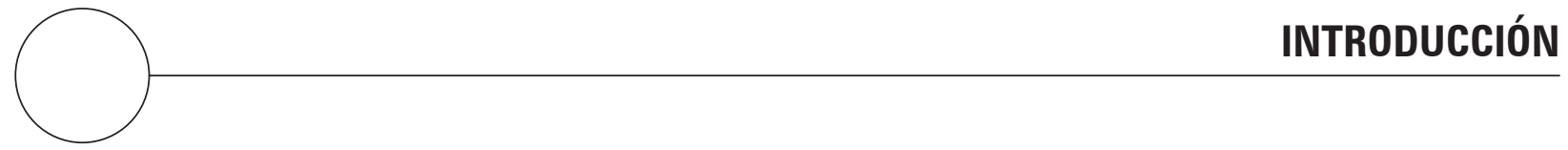

La producción agrícola depende de tecnologías y practicas dirigidas a obtener un mayor rendimiento, tal como lo es el uso de fertilizantes. Los cultivos de trigo, arroz y maíz consumen más del $50 \%$ de los fertilizantes de uso mundial, sin embargo, a pesar del elevado uso de los mismos se obtienen productividades bajas, lo que sugiere que los fertilizantes son usados en forma ineficiente (FAO, 2010).

El consumo mundial de fertilizantes $\left(\mathrm{N}, \mathrm{P}_{2} \mathrm{O}_{5}, \mathrm{~K}_{2} \mathrm{O}\right)$ en el año 2012 fue de 180,1 millones de toneladas, y se prevé un incremento anual del 1,9\% hasta 2018, siendo los fertilizantes nitrogenados los de mayor demanda con el 61\% del consumo global (FAO, 2015). Por otra parte, este aumento de la fertilización nitrogenada incrementa la emisión de óxido nitroso (Arunrat y Pumijumnong, 2017). En este sentido, el IDEAM (2016) reporta para Colombia qué de la emisión total de GEI en millones de toneladas (258,8 Mton $\mathrm{CO}_{2} \mathrm{Eq}$ ) a nivel nacional, el sector agropecuario ocupa el segundo lugar con $26 \%$, de los cuales aproximadamente el $18,5 \%$ corresponde a $\mathrm{N}_{2} \mathrm{O}$, y a su vez el $2,5 \%$ es originado por uso de fertilizantes; el departamento del Valle del Cauca ocupa el cuarto lugar a nivel nacional con 16,5 Mton $\mathrm{CO}_{2} \mathrm{Eq}$, de las cuales el 23,3\% se originan en el sector económico agropecuario y el $4,77 \%$ corresponde a $\mathrm{N}_{2} \mathrm{O}$.

El óxido nitroso presenta un potencial de calentamiento de 298 veces más que una molécula de dióxido de carbono en 100 años (IPCC, 2007), siendo considerado uno de los principales gases responsables del calentamiento global, y por lo tanto es necesario implementar un apropiado manejo de fertilizantes y prácticas que contribuyan a su disminución. En este orden de ideas, la zeolita surge como alternativa en el uso eficiente de nutrientes y reducción de la emisión de $\mathrm{N}_{2} \mathrm{O}$. La clinoptilolita es una de las 48 especies de zeolita natural más abundante y utilizada para retener el nitrógeno en el suelo (He et al., 2002). Esta arcilla natural posee alta capacidad de intercambio catiónico y gran afinidad por iones amonio $\left(\mathrm{NH}_{4}{ }^{+}\right)$(Inglezakis et al., 2004). Presenta influencia en el rendimiento, absorción de nitrógeno y aumento de materia seca (Malekian et al., 2011), así mismo, Torma et al. (2014) sugieren su importancia en forma positiva en la nutrición de plantas. Por lo tanto, su utilización puede mejorar el uso eficiente de nutrientes, lo cual tendrá una influencia positiva sobre la producción vegetal y la mitigación de la contaminación ambiental como lo sugieren Bolado et al. (2003), que finalmente contribuirá con la sustentabilidad de los agroecosistemas.

Las investigaciones muestran la importancia de la implementación de zeolita en diversos sistemas de producción agrícola, Flores et al. (2007) muestran un efecto positivo para el cultivo de avena sobre la producción de biomasa seca; Osuna et al. (2012) afirman que mejora el uso eficiente de los fertilizantes nitrogenados, fosfóricos y rendimiento del trigo. Chibsa et al. 
(2017) recomiendan la clinoptilolita para aumentar la eficiencia de absorción de agua, nutrientes y a su vez disminuir la lixiviación de nitratos. En tomate, Afrous y Goudarzi (2015) mencionan que el uso de zeolita contribuyó con un mejor uso eficiente de fertilizantes y agua. Obregón et al. (2016) obtuvieron mayores rendimientos en maíz con aplicaciones de zeolita, mostrando mayor eficiencia en el uso de nitrógeno. Sin embargo, estas investigaciones se refieren en su gran mayoría al uso eficiente de nitrógeno por efecto de la zeolita y no incluyen análisis rigurosos con respecto a los demás nutrientes ni tampoco para gases efecto invernadero como $\mathrm{N}_{2} \mathrm{O}$.

La mayoría de las investigaciones son internacionales y pocas en el ámbito nacional, así mismo, es relevante considerar que la dinámica suelo-clinoptilolita-nutrientes es variable, ya que depende del tipo y manejo del cultivo, dosis de zeolita y de las propiedades físicas y químicas del suelo, entre otras (Kolyagin y Karasev, 1999). Por tal motivo, es trascendental evaluar la dosis de zeolita específica para cada suelo y genotipo. En este orden de ideas, esta investigación tuvo como objetivo determinar la influencia de la zeolita en la emisión de óxido nitroso y en el uso eficiente de nutrientes (N-P-K) en términos de eficiencia agronómica (EA) y eficiencia de recuperación del fertilizante (ERF) para el hibrido GSS-4644 de maíz.

\section{MATERIALES Y MÉTODOS}

La investigación se realizó en el Centro Experimental de la Universidad Nacional de Colombia sede Palmira (CEUNP), ubicado geográficamente a $3^{\circ} 25^{\prime} 34^{\prime \prime} \mathrm{N}$ y $76^{\circ} 25^{\prime} 53^{\prime \prime}$ W, a $980 \mathrm{msnm}$, en el municipio de Candelaria (corregimiento El Carmelo), departamento del Valle del Cauca. La zona presenta temperatura media del aire $24^{\circ} \mathrm{C}$, humedad relativa $69 \%$ y precipitación promedio anual de $1.406 \mathrm{~mm}$.

El experimento se llevó a cabo en suelo clasificado como Ustic Epiaquert (IGAC, 2006), se muestreó a 20 $\mathrm{cm}$ de profundidad y se caracterizó en el laboratorio de suelos del Centro Internacional de Agricultura Tropical (CIAT) siguiendo metodologías estándar (Mckean, 1993); densidad aparente por el método del núcleo o cilindro, textura por el método de la pipeta, densidad real por picnómetro, porosidad total por cilindro, $\mathrm{pH}$ mediante potenciómetro (1:1), materia orgánica por Walkey-Black, fósforo por el método Olsen, capacidad de intercambio catiónico por acetato de amonio $\mathrm{pH} 7,0$, como también, $\mathrm{NH}_{4}{ }^{+}$por reacción de Berthelot, a los 0 y 76 d después de siembra; los datos se interpretaron con valores referencia del Instituto Colombiano Agropecuario (ICA, 1992).

Los tratamientos diseñados incluyen un testigo y variaciones de zeolita en dosis de 0,30, 60 y 90\% con respecto a la dosis de nitrógeno (Tab. 1); la dosis de nutrientes se precisó de acuerdo a Narváez (2008), además, se tuvo en cuenta el contenido de nutrientes del suelo y las eficiencias de uso sugeridas por Dobermann (2007) para N, P y K de 63, 47 y 63\% respectivamente.

Tabla 1. Descripción de los tratamientos.

\begin{tabular}{|c|c|c|c|c|}
\hline \multirow{2}{*}{ Tratamiento } & \multicolumn{5}{|c|}{ Dosis $\left(\mathrm{kg} \mathrm{ha}^{-1}\right)$} \\
\cline { 2 - 5 } & $\mathbf{Z}$ & $\mathrm{N}$ & $\mathrm{P}_{2} \mathrm{O}_{5}$ & $\mathrm{~K}_{2} \mathrm{O}$ \\
\hline T0 & 0 & 0 & 0 & 0 \\
\hline T1 & 0 & 236 & 75 & 192 \\
\hline T2 & 70 & 236 & 75 & 192 \\
\hline T3 & 140 & 236 & 75 & 192 \\
\hline T4 & 210 & 236 & 75 & 192 \\
\hline
\end{tabular}

Z: zeolita; N: nitrógeno; $\mathrm{P}_{2} \mathrm{O}_{5}$ : fósforo; $\mathrm{K}_{2} \mathrm{O}$ : potasio.

La fuente de nitrógeno empleada fue urea (46-00-00) marcada con el isótopo ${ }^{15} \mathrm{~N}$, para fósforo se utilizó agro K (00-93-52) y el potasio restante se suplió con cloruro de potasio (00-00-60).

El diseño experimental consistió en bloques completos al azar (BCA) con cinco tratamientos y cuatro repeticiones, para un total de veinte unidades experimentales (UE). La UE se conformó por una parcela de $28,8 \mathrm{~m}^{2}(6 \times 4,8 \mathrm{~m})$ con 180 plantas sembradas a $0,2 \times 0,8 \mathrm{~m}$, lo que equivale a 62.500 plantas/ha. Dentro de cada parcela fue delimitada un área central de $7,68 \mathrm{~m}^{2}$ correspondiente a 48 plantas, en la cual se incorporó el nitrógeno marcado con el isótopo ${ }^{15} \mathrm{~N}$ y se instaló la cámara estática cerrada (CSC) descrita por Ferreira (2008), para el muestreo de óxido nitroso $\left(\mathrm{N}_{2} \mathrm{O}\right)$, así, un anillo de $8 \mathrm{~cm}$ de altura y $25 \mathrm{~cm}$ de diámetro permaneció fijo en el terreno durante todo el ciclo experimental.

Las cámaras se muestrearon a los 15, 25, 35, 46, 56, 65 y 76 días después de la siembra (dds), tomando cuatro submuestras en intervalos de 0,10,20 y $30 \mathrm{~min}$, siempre a la misma hora de la mañana (Rondón, 2000). Al mismo tiempo, se midió la temperatura con termómetro adaptado a la cámara y la humedad gravimétrica del suelo en base seca, para calcular adecuadamente el 
flujo gaseoso. El flujo de $\mathrm{N}_{2} \mathrm{O}$ se determinó mediante cromatografía de gases en el Laboratorio de Servicios Ambientales del CIAT (Cali) y se calculó el Potencial de Calentamiento Global (PCG) para los 76 d acumulados de evaluación por cada tratamiento siguiendo metodología del CIAT (2009), de acuerdo con esto, los valores positivos del flujo de GEI indican una emisión desde el suelo hacia la atmósfera y los negativos, emisión desde la atmósfera al suelo.

El material vegetal evaluado fue el híbrido de maíz dulce GSS-4644, el cual, cinco días después de la germinación fue sembrado en campo. La dosis de fertilización se fraccionó en dos épocas de acuerdo con los estadios de crecimiento sugeridos por Bolaños y Edmeades (1992): 40\% en la primera aplicación (15 dds), la cual corresponde al estadio vegetativo V6 (periodo de rápida elongación del tallo, siendo visible el cuello de la hoja 6) y el $60 \%$ restante ( $35 \mathrm{dds}$ ), coincide con el comienzo del periodo reproductivo, en el estadio vegetativo VT (visible la última rama de la espiga). El $100 \%$ de la zeolita se incorporó al suelo junto con la primera aplicación de fertilizante y la aplicación del isótopo ${ }^{15} \mathrm{~N}$ se realizó en las mismas proporciones de nitrógeno requerido, en este sentido, el fertilizante urea se enriqueció al 2,5\% ${ }^{15} \mathrm{~N}$ átomos en exceso en cada aplicación y se aplicaron diluidos a partir de un volumen de mezcla en agua de $10 \mathrm{~mL}$ por planta (Urquiaga y Zapata, 2000).

El rendimiento de grano estandarizado al 14\% de humedad se estimó a partir de 30 plantas de cada parcela (Peréz, 2001). Para los análisis de tejido se colectaron 22 mazorcas por tratamiento y réplica, después se determinó la concentración de fósforo y potasio en base seca $\left(550^{\circ} \mathrm{C}\right)$ por colorimetría y absorción atómica respectivamente (Mckean, 1993), como también, el isotopo ${ }^{15} \mathrm{~N}$ por espectrofotometría de masas en la Universidad de Davids en California, Estados Unidos, así, con los datos de $\mathrm{N}$ total (\%) y exceso de ${ }^{15} \mathrm{~N}$ (At-\%) se calculó el nitrógeno derivado del fertilizante (Nddf) (Acón-Ho et al., 2013). Además, se estimó para N-P-K la eficiencia agronómica (EA) y eficiencia de recuperación del fertilizante (ERF) de acuerdo con las ecuaciones propuestas por Baligar et al. (2001).

Los datos obtenidos fueron sometidos a análisis de varianza (ANDEVA) para determinar diferencias entre las variables de respuesta teniendo como fuente de variación, bloque, tratamiento y la interacción bloque $\times$ tratamiento, posteriormente los promedios se compararon mediante la prueba de Tukey $(P \leq 0,05)$; se realizaron correlaciones de Pearson entre zeolita, flujo acumulado de $\mathrm{N}_{2} \mathrm{O}$, PCG, EA y ERF, utilizando el programa Statistical Analysis System (SAS).

\section{RESULTADOS Y DISCUSIÓN}

Los análisis físico y químico del suelo mostraron condiciones no limitantes para el cultivo de maíz de acuerdo al INIA (Villaseca y Novoa, 1987), en tanto, el suelo presentó textura franco arcilloso (arena 32,98\%, arcilla $33,42 \%$ y limo $33,6 \%$ ), densidad aparente de $1,57 \mathrm{Mg} \mathrm{m}^{-3}$, porosidad total $37,6 \%, \mathrm{pH}$ neutro de 7,22 , bajo contenido de materia orgánica (1,45\%), fósforo $(82,08 \mathrm{ppm})$, potasio $\left(0,67 \mathrm{cmol} \mathrm{kg}^{-1}\right), 3,87 \mathrm{ppm}$ de $\mathrm{NH}_{4}{ }^{+}$inicial (0 dds), 4,5 ppm $\mathrm{NH}_{4}{ }^{+}$final (76 dds), y alta capacidad de intercambio catiónico $(39,34 \mathrm{cmol}$ $\mathrm{kg}^{-1}$ ).

\section{Influencia de la zeolita en la emisión de óxido nitroso $\left(\mathrm{N}_{2} \mathrm{O}\right)$}

La emisión de óxido nitroso a partir de los 15 d después de la siembra hasta los 76 dds mostró diferencias altamente significativas $(P \leq 0,01)$ por efecto del bloque, tratamiento e interacción bloque $\times$ tratamiento (Tab. 2).

Tabla 2. Análisis de varianza para óxido nitroso con respecto a los días después de siembra en maíz dulce.

\begin{tabular}{|l|c|c|c|c|c|c|c|}
\hline \multirow{2}{*}{$\begin{array}{c}\text { Fuente de } \\
\text { variación }\end{array}$} & \multicolumn{6}{|c|}{ Días después de siembra } \\
\cline { 2 - 8 } & 15 & 25 & 35 & 46 & 56 & 65 & 76 \\
\hline Bloque & $* *$ & $* *$ & $* *$ & $* *$ & $* *$ & $* *$ & $* *$ \\
\hline Tratamiento & $* *$ & $* *$ & $* *$ & $* *$ & $* *$ & $* *$ & $* *$ \\
\hline Bloque $\times$ tratamiento & $* *$ & $* *$ & $* *$ & $* *$ & $* *$ & $* *$ & $* *$ \\
\hline
\end{tabular}

** Diferencias altamente significativas $(P \leq 0,01)$.

Las emisiones de óxido nitroso en los siete muestreos evidencian por una parte la influencia de la fertilización en la emisión de este gas efecto invernadero, dado que la menor emisión se presentó en el T0 (testigo sin N-P-K) y la mayor en el T1 (testigo con N-P-K); por otra parte, a partir del T3 (Z140 con N-P-K) se percibe influencia de la zeolita mitigando el flujo de óxido nitroso. En este sentido, para 25, 35, 46 y 65 dds es contundente la influencia de la zeolita, sin embargo, 15, 56 y 76 dds el efecto fue diferente, el valor negativo de la emisión de $\mathrm{N}_{2} \mathrm{O}$ a los 56 dds en el tratamiento T0 $\left(-3,1 \mathrm{~g} \mathrm{ha}^{-1} \mathrm{~d}^{-1}\right)$ indica que el suelo se comportó como un sumidero del gas, igual sucede para los $76 \mathrm{dds}$ en el T0 (-2,6 $\left.\mathrm{g} \mathrm{ha}^{-1} \mathrm{~d}^{-1}\right)$ y T4(-1,9 $\left.\mathrm{g} \mathrm{ha}^{-1} \mathrm{~d}^{-1}\right)$ (Fig. 1), el cual 
se atribuye a las condiciones de humedad del suelo como lo sugieren Bergstrom et al.(2001).

El rendimiento y potencial de calentamiento global para óxido nitroso mostraron diferencias altamente significativas $(P \leq 0,01)$ por bloque, tratamiento $y$ por la interacción bloque $\times$ tratamiento. La menor emisión acumulada se presentó en el T0 (1,51 $\mathrm{g} \mathrm{ha}^{-1}$ $\left.\mathrm{d}^{-1}\right)$ y por consiguiente el menor valor de PCG $(708,8$ $\mathrm{kg} \mathrm{CO}_{2}$-Eq ha $\left.{ }^{-1} \mathrm{año}^{-1}\right)$, como también, el menor rendimiento (842 kg ha-1). El mayor PCG se obtuvo en el T1 $\left(6.856,5 \mathrm{~kg} \mathrm{CO}_{2}-\mathrm{Eq} \mathrm{ha}{ }^{-1}\right.$ año-1) y el mayor rendimiento en el T4 (8.627 kg ha-1), los cuales disminuyeron y aumentaron respectivamente con el incremento de la cantidad de zeolita (Fig. 2) como lo sugiere Torma et al. (2014).

En este sentido, el PCG para T0 representa el 10,34\% con respecto al 100\% del T1, y a partir del T2 empieza a disminuir el PCG en 33\%, luego un 14\% en el T3 y finalmente, $2,9 \%$ en el $\mathrm{T} 4$, lo que sugiere que la eficiencia de la zeolita disminuye a mayor cantidad de kilogramos, en tanto, a partir de $70 \mathrm{~kg} \mathrm{ha}^{-1}$ de zeolita empieza a disminuir el PCG hasta la dosis de $210 \mathrm{~kg}$ $h^{-1}{ }^{-1}$ cada vez en menor proporción, resultados similares obtuvieron González et al. (2012).

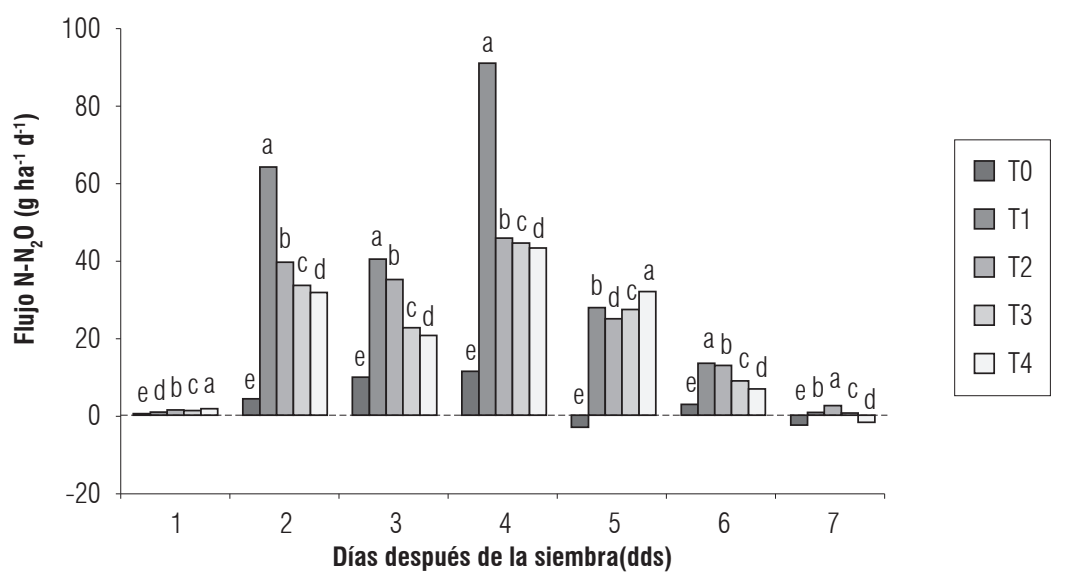

Figura 1. Flujo de óxido nitroso por cada tratamiento en diferentes días después de la siembra. Promedios con letras distintas indican diferencia significativa según la prueba de Tukey $(P \leq 0,05)$.

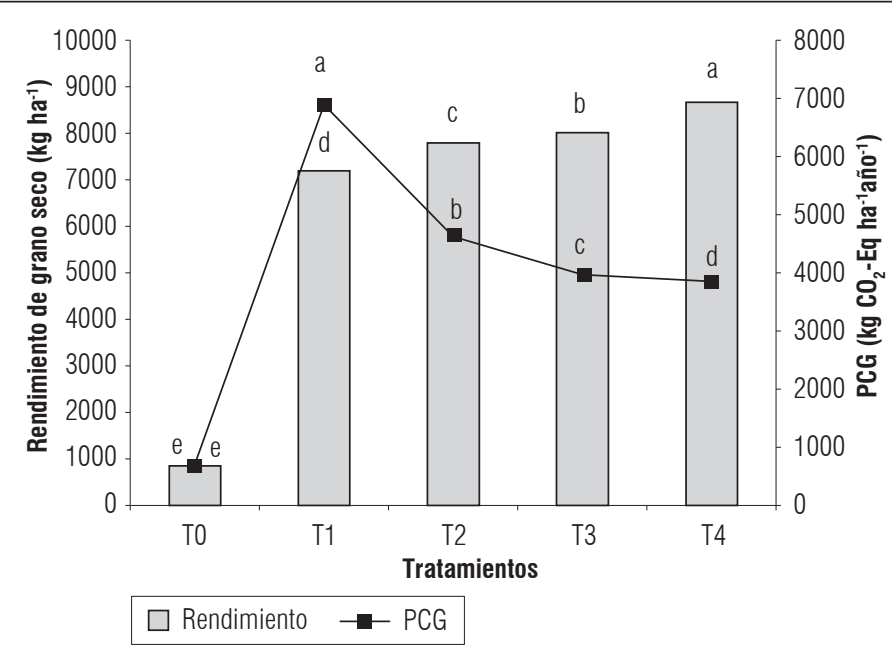

Figura 2. Potencial de calentamiento global del óxido nitroso y rendimiento de grano seco de maíz dulce por cada tratamiento. Promedios con letras distintas indican diferencia significativa según la prueba de Tukey $(P \leq 0,05)$. 
Los mayores rendimientos se presentaron en aquellos tratamientos con mayor cantidad de zeolita en el siguiente orden: T4 (8.627 kg ha-1), T3 (7.980 $\left.\mathrm{kg} \mathrm{ha}^{-1}\right)$, T2 (7.754 kg ha-1), T1 (7.156 kg ha-1) y T0 (842 $\left.\mathrm{kg} \mathrm{ha}^{-1}\right)$, siendo superiores con respecto a Gordon-Mendoza et al. (2016). La aplicación de fertilizante contribuyo en mayor rendimiento en el T1 con respecto al T0 como lo sugieren Romero-Lozada et al. (2017), así mismo, la zeolita promovió incrementos en el rendimiento desde T2 hasta T4, siendo similares con los resultados de Osuna et at. (2012), y superiores comparados con los valores reportados por Obregón et al. (2016).

\section{Influencia de la zeolita en el uso eficiente de nutrientes}

El análisis de varianza mostro diferencias altamente significativas por efecto de bloque, tratamiento e interacción bloque $\times$ tratamiento para eficiencia agronómica de nitrógeno (EAN), fósforo (EAP) y potasio (EAK), como también, para la eficiencia de recuperación del fertilizante (ERF) para N-P-K. Por tratamiento, los mayores valores de eficiencia agronómica se presentaron en el T4, seguido T3, T2 y por último T1 para N-P-K, siendo evidente la influencia de la zeolita sobre la EAN, es decir, por cada kg de nitrógeno aplicado al suelo en T4 el rendimiento se aumentó en $32,99 \mathrm{~kg}$ de grano seco, en T3 se aumentó $30,25 \mathrm{~kg}$, $29,29 \mathrm{~kg}$ en T2 y 26,75 en T1; la EAP en T4 aumentó el rendimiento en 103,83 kg, en T3 95,18 kg, T2 92,18 kg y en T1 84,20 kg; así mismo, la EAK fue 40,48 kg, $37,18 \mathrm{~kg}, 35,93 \mathrm{~kg}$ y $32,89 \mathrm{~kg}$ para T4, T3, T2 y T1, respectivamente (Fig. 3). Estos resultados superan los reportados por Obregon et al. (2016), mostrando una tendencia a lograr mayores incrementos en la producción por $\mathrm{kg}$ de nutriente aplicado en aquellos tratamientos que contienen más zeolita.

En relación a la eficiencia de recuperación de nitrógeno del fertilizante (ERNF), se observa que los mayores valores se muestran en el T3, seguido T2, T4 y por último el T1, lo que significa que del 100\% de nitrógeno aplicado al suelo se recuperó de mayor a menor, el $51,23 \%$ en $\mathrm{T} 3,49,28 \%$ en $\mathrm{T} 2,47,65 \%$ en T4 y $35,49 \%$ en el T1(Fig. 4), estos valores son similares con los reportados por Obregón et al. (2016). En tanto, las pérdidas de nitrógeno superan más del $50 \%$ asociadas a volatilización, denitrificación y lixiviación (Brye et al., 2001), las cuales son normales para el trópico (Stipp y Prochnow, 2008), no obstante, es evidente la influencia benéfica de la zeolita.

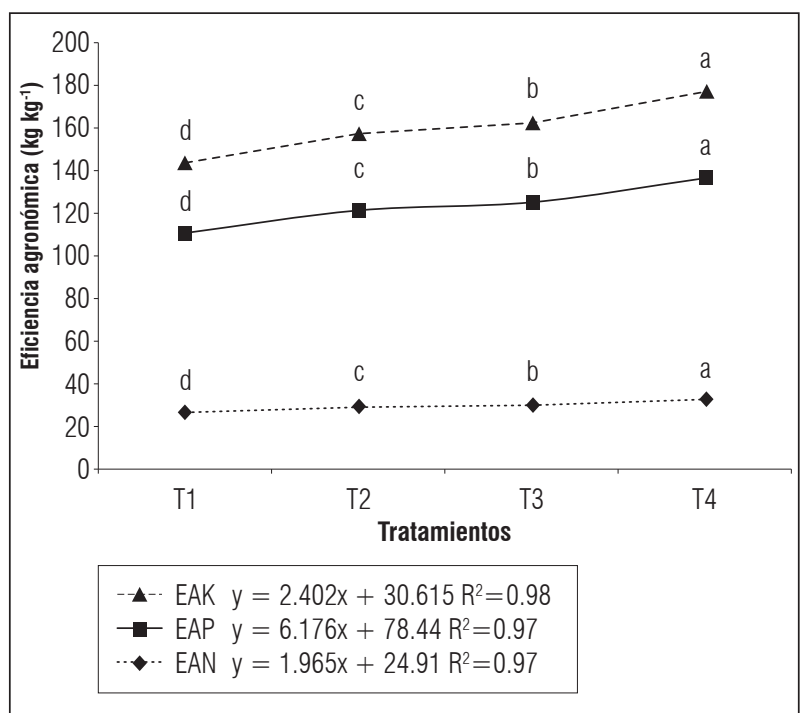

Figura 3. Eficiencia agronómica para nitrógeno, fósforo y potasio. Promedios con letras distintas indican diferencia significativa según la prueba de Tukey $(P \leq 0,05)$.

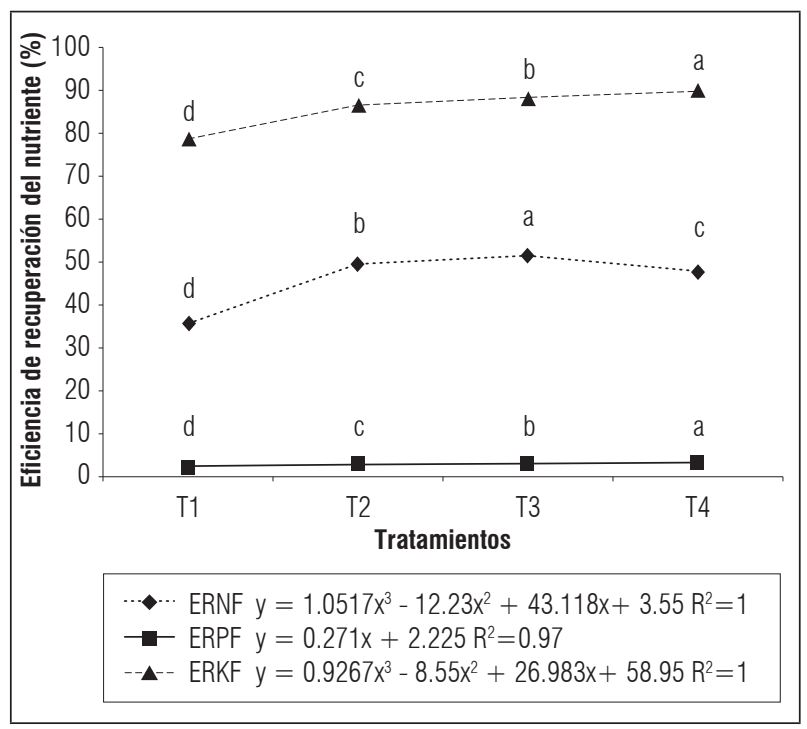

Figura 4. Eficiencia de recuperación del nutriente para nitrógeno, fósforo y potasio por tratamiento. Promedios con letras distintas indican diferencia significativa según la prueba de Tukey $(P \leq 0,05)$.

Es importante resaltar que mayores dosis de zeolita pueden retener el nitrógeno en forma de $\mathrm{NH}_{4}{ }^{+}$en los sitios de intercambio, con lo cual su disponibilidad para la planta se limita, esto explica la baja eficiencia en T4 con mayor cantidad de zeolita, corroborado con 
el mayor contenido en el suelo de amonio (4,5 ppm) al final del experimento, dada la afinidad por los iones de amonio de la zeolita como lo sugieren Inglezakis et al. (2004). Por lo tanto, es claro que la dosis de zeolita unida al fertilizante influencia la sincronización entre la absorción de la planta de maíz y la liberación de nitrógeno del sistema suelo-fertilizante-zeolita como lo referencian Civeira et al. (2011).

La eficiencia de recuperación de fósforo del fertilizante muestra los mayores valores en T4, seguido T3, T2 y por último T1, en efecto, del 100\% de fósforo aplicado al suelo se recuperó de mayor a menor, el 3,29\% en T4, 3,04\% en T3, 2,82\% en T2 y 2,46\% en el T1; la ERKF presentó similar comportamiento con mayores valores, en este sentido, del $100 \%$ de potasio aplicado al suelo se recuperó 89,$39 ; 87,97 ; 86,13$ y $78,31 \%$ para T4, T3, T2 y T1 respectivamente (Fig. 4), estas eficiencias se consideraron bajas para fósforo y altas para potasio comparadas con Puentes et al. (2014).

Las correlaciones entre zeolita y $\mathrm{N}_{2} \mathrm{O}$ acumulado en los 76 dds, PCG, EA y ERF para nitrógeno isotópico, fósforo y potasio son altamente significativas, mostrando que con mayor cantidad de zeolita se disminuye la emisión de óxido nitroso y el potencial de calentamiento global, en contraste, se favorece el uso eficiente de N-P-K (Tab. 3). Similar resultado obtuvieron Valerio et al. (2016), mostrando que la zeolita disminuye los riesgos de contaminación del aire, asociados a la volatilización del precursor de óxido nitrógeno.

\section{CONCLUSIONES}

La zeolita influencia la emisión de óxido nitroso y uso eficiente de nutrientes, de tal manera que a partir de $70 \mathrm{~kg} \mathrm{ha}^{-1}$ de clinoptilolita se reduce el flujo de óxido nitroso hacia la atmosfera en un 33\% y mejora en 9\% la eficiencia de uso para N-P-K en términos de eficiencia agronómica y en $22 \%$ la eficiencia de recuperación del fertilizante; no obstante, la emisión de $\mathrm{N}_{2} \mathrm{O}$ se reduce y la eficiencia de uso de N-P-K aumenta hasta la dosis de $210 \mathrm{~kg} \mathrm{ha}^{-1}$ de zeolita, excepto para la recuperación de nitrógeno dada la afinidad por los iones de amonio. En este orden de ideas, la zeolita mitiga las emisiones de óxido nitroso y mejora el uso eficiente de nutrientes, sin embargo, es conveniente realizar investigaciones específicas para cada zona dada la variabilidad de las condiciones edafoclimáticas y diversidad de cultivos.

\section{AGRADECIMIENTOS}

Los autores agradecen al Grupo de Investigación: "Uso y Manejo de Suelos y Aguas con énfasis en Degradación de Suelos" y al Centro experimental de la Universidad Nacional de Colombia, sede Palmira (CEUNP), como también, a la Corporación Autónoma Regional del Valle del Cauca (CVC) quien financió la presente investigación.

Conflicto de intereses: el manuscrito fue preparado y revisado con la participación de los autores, quienes declaran no tener algún conflicto de interés que coloquen en riesgo la validez de los resultados aquí presentados.

\section{REFERENCIAS BIBLIOGRÁFICAS}

Acón-Ho J., U.C. Cervantes y R. WingChing-Jones. 2013. Recuperación del ${ }^{15} \mathrm{~N}$ en la planta de banano y en el suelo de áreas con origen sedimentario. Agron. Mesoam. 24(1), 71-81.

Afrous, A. y S. Goudarzi. 2015. The effect of different types of zeolite on drain water volume and nitrate leaching under tomato cultivated. J. Scientific Res. Develop. 2(1), 56-58.

Arunrat, N. y N. Pumijumnong. 2017. Practices for reducing greenhouse gas emissions from rice production in Northeast Thailand. Agric. 7(1), 4. Doi: 10.3390/ agriculture7010004

Baligar, V.C., N.K. Fageria y Z.L. He. 2001. Nutrient use efficiency in plants. Comm. Soil Sci. Plant Anal. 32(7), 921-950. Doi: 10.1081/CSS-100104098

Bergstrom, D.W., M. Tenuta y E.G. Beauchamp. 2001. Nitrous oxide production and flux from soil under sod following application of different nitrogen fertilizers. Comm. Soil Sci. Plant Anal. 32(3-4), 553-570. Doi: 10.1081/CSS-100103028

Tabla 3. Correlaciones entre zeolita y variables de respuesta para el hibrido GSS-4644 de maíz dulce.

\begin{tabular}{|c|c|c|c|c|c|c|c|c|}
\hline Híbrido & $\mathrm{N}_{2} \mathrm{O}$ & PCG & EAN & EAP & EAK & ERNF & ERPF & ERKF \\
\hline GSS-4644 & $-0.668^{* *}$ & $-0,728^{* *}$ & $0,967^{* *}$ & $0,965^{* *}$ & $0,965^{* *}$ & $0,556^{* *}$ & $0,876^{* *}$ & $0,638^{* *}$ \\
\hline
\end{tabular}

** Correlación altamente significativa al nivel 0,01 
Bolado, S.A., M. Alonso y J. Alvarez-Benedi. 2003. Caracterización de procesos acoplados de adsorción, transformación y volatilización de $\mathrm{N}$ en suelos fertilizados con urea. pp. 185-192. En: Alvarez-Benedi, J. y R. Marinero (eds.). Estudios de la zona no saturada del suelo. Vol. VI. Instituto Tecnológico Agrario de Castilla y León (ITA), Valladolid, España.

Bolaños, J. y G. Edmeades. 1992. La fenología del maíz. Síntesis de resultados experimentales del PRM 4, 251-261.

Brye, K.R., J.M. Norman, L.G. Bundy y S.T. Gower. 2001. Nitrogen and carbon leaching in agroecosystems and their role in denitrification potential. J. Environ. Qual. 30, 58-70. Doi: 10.2134/jeq2001.30158x

Chibsa, T., H. Gebrekidan, K. Kibret y T. Debele. 2017. Response of durum weat to clinoptilolite zeolite and nitrogen fertilizer rates on cambisols of bale highlands, Southeastern Ethiopia. World Appl. Sci. J. 35(1), 1826. Doi: 10.5829/idosi.wasj.2017.18.26

CIAT. 2009. Presentación de toma de muestra, análisis y cálculo del potencial de calentamiento global (PCG) en sistemas agroforestales y silvopastoriles. Manual de análisis de suelos y tejido vegetal. Centro Internacional de Agricultura Tropical, Cali, Colombia.

Civeira, G. y M.B. Rodríguez. 2011. Nitrógeno residual y lixiviado del fertilizante en el sistema suelo-planta-zeolita. Ci. Suelo. 29(2), 285-294.

Dobermann, A. 2007. Nutrient use efficiency: measurement and management. pp. 1-21. En: Proc. International Fertilizer Industry Association (IFA). Workshop on Fertilizer Best Management Practices. Bruselas, Bélgica.

FAO. 2010. Estrategias en materia de fertilizantes. Food and Agriculture Organization of the United Nations, Roma, Italia.

FAO. 2015. Estimación de emisiones de gases de efecto invernadero en la agricultura. Un manual para abordar los requisitos de los datos para los países en desarrollo. Food and Agriculture Organization of the United Nations, Roma, Italia.

Ferreira, O.I. 2008. Flujos de gases de efecto invernadero, potencial de calentamiento global y evaluación de emergía del sistema agroforestal Quesungual en el sur de Lempira, Honduras. Tesis de maestría. Programa de Maestría en Ciencias Agrarias, Universidad Nacional de Colombia, Palmira, Colombia.

Flores, A., A. Galvis, T. Hernández, F. De León y F. Payán. 2007. Efecto de la adición de zeolita (clinoptilolita y mordenita) en un andosol sobre el ambiente químico edáfico y el rendimiento de avena. Interciencia 32(10), 692-696.

González, M., N. Gómez, J. Muñiz, F. Valencia, D. Gutiérrez y H. Figueroa. 2012. Rendimiento del maíz de riego tratado con zeolita más fertilizantes en el estado de Guerrero. Rev. Mex. Cienc. Agríc. 3(6), 1129-1144.
Gordon-Mendoza R., J.E. Franco-Barrera, J.E. Villarreal-Núñez y T.J. Smith. 2016. Manejo de la fertilización fosforada en el cultivo de maíz, el ejido, panamá 2004-2013. Agron. Mesoam. 27(1), 95-108. Doi: 10.15517/am.v27i1.21889

He, Z.L., D.V. Calvert, A.K. Li, and D.J. Alva-Banks. 2002. Clinoptilolite zeolite and cellulose amendments to reduce ammonia volatilization in a calcareous sandy soil. Plant Soil. 247, 253-260.

ICA (Instituto Colombiano Agropecuario). 1992. Fertilización en diversos cultivos. Quinta Aproximación. Manual de Asistencia Técnica No. 25. Centro de Investigación Tibaitatá, Mosquera, Colombia.

IDEAM (Instituto de Hidrología, Meteorología y Estudios Ambientales). 2016. Inventario nacional y departamental de gases efecto invernadero - Colombia. $3^{\text {a }}$ Comunicación nacional de cambio climático. Bogotá, Colombia.

IGAC. 2006. Suelos de Colombia. Instituto Geográfico Agustin Codazzi. Subdirección de Agrología, Bogotá, Colombia.

Inglezakis, V., M. Loizidou y H. Grigoropoulou. 2004. Ion exchange studies on natural and modified zeolites and the concept of exchange site accessibility. J. Colloid Interface Sci. 275(2), 570-576. Doi: 10.1016/j. jcis.2004.02.070

IPCC (Intergovernmental Panel on Climate Change). 2007. Climate change 2007: Impacts, adaptation and vulnerability. pp. 130-234. Fourth Assessment Report on Climate Change. Cambridge University Press, Cambridge, UK.

Kolyagin, S. y O.A. Karasev. 1999. Root nutrition and the quality of sugarbeet. Sakharnaya Svekla 6, 11-12.

Mckean, S.J. 1993. Manual de analisis de suelos y tejido vegetal: una guía teórica y práctica de metodologías. Documento de trabajo No. 129. Laboratorio de servicios analíticos, Centro Internacional de Agricultura Tropical (CIAT), Cali, Colombia.

Malekian, R., J. Abrdi-Koupai y S. Eslamian. 2011. Influences of clinoptilolite and surfactant-modified clinoptilolite zeolite on nitrate leaching and plant growth. J. Hazard. Mater. 185(2-3), 970-976. Doi: 10.1016/j. jhazmat.2010.09.114

Narváez, C.M., M. Sánchez y F.J.C. Menjívar. 2010. Efecto de la aplicación de vinazas en las propiedades físicas y la actividad de deshidrogenasas en suelos cultivados con maíz dulce (Zea mays L.). Acta Agron. 59(2), 211-217.

Obregón, N., J.E. Díaz, M.C. Daza y H.F. Aristizabal. 2016. Efecto de la aplicación de zeolita en la recuperación de nitrógeno y el rendimiento de maíz. Acta Agron. 65(1), 24-30.

Osuna, C.E.S., A.M. Ramírez, R. Paredes-Melesio, R.J.S. Padilla y G.A.D. Báez. 2012. Eficiencia de la zeolita como 
aditivo de la urea e inoculación micorrizica en el cultivo de trigo. Rev. Mex. Cienc. Agríc. 3(6), 1101-1113.

Pérez, C.J.P. 2001. Metodología para la evaluación de cosechas de maíz en parcelas comerciales. Instituto Nacional de Investigaciones Forestales, Agrícolas y Pecuarias. Pachuca, Hidalgo, México

Puentes, P.Y.J., F.J.C. Menjivar y H.F. Aránzazu. 2014. Eficiencias en el uso de nitrógeno, fósforo y potasio en clones de cacao (Theobroma cacao L.). Bioagro. 26, 99-106.

Romero-Lozada M., Y.J. Puentes-Páramo y J.C. Menjivar-Flores. 2017. Extraction of mineral nutrients in pepper (Capsicum sp.) leaves and fruits and its influence on yield. Rev. Colomb. Cienc. Hort. 11(1), 112-119. Doi: 10.17584/rcch.2017v11i1.5809

Rondón, M. 2000. Land use and balances of greenhouse gases in Colombian Tropical Savannas. Ph.D. thesis. Cornell University, New York, USA.
Torma, S., J. Vilcek, P. Adamisin, E. Huttmanova y O. Hronec. 2014. Influence of natural zeolite on nitrogen dynamics in soil. Turk. J. Agric. For. 38, 739-744. Doi: 10.3906/tar-1311-1313

Urquiaga, D. y F. Zapata. 2000. Metodologías isotópicas para estudios de la eficiencia de la fertilización nitrogenada y otros procesos del ciclo del N. pp. 25-29. En: Urquiaga, S. y F. Zapata (eds.). Manejo eficiente de la fertilización nitrogenada de cultivos anuales en America Latina y el Caribe. Génesis, Embrapa, Porto Alegre, Brasil.

Valerio, L.S.G., L.R. Quintero, C.G. A.J. Baca y L.A.G. Quispe. 2016. Captación de amonio en zeolita al incubar gallinaza y residuos de codorniz. Terra Latinoam. 34, 201-206.

Villaseca, C.S. y S.-A.R. Novoa. 1987. Requerimiento: de suelo y clima del maíz. IPA La Platina 43, 38-40. 